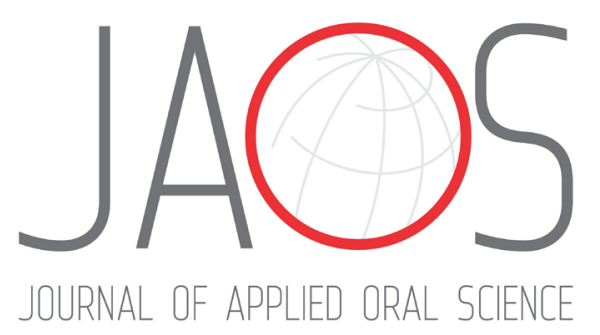

\title{
The effect of reduced glutathione on the toxicity of silver diamine fluoride in rat pulpal cells
}

\section{Abstract}

Seunggun KIM ${ }^{1}$
Mohannad NASSAR ${ }^{2}$
Yukihiko TAMURA ${ }^{3}$
Noriko HIRAISHI ${ }^{1}$
Ahmed JAMLEH ${ }^{4,5}$
Toru NIKAIDO
Junji TAGAMI

Submitted: October 17, 2020 Modification: January 7, 2021 Accepted: January 15, 2021

Corresponding address: Noriko Hiraishi Tokyo Medical and Dental University Graduate School of Medical and DentalSciences Department of Cariology and Operative

Dentistry - Tokyo - Japan.

e-mail: hir

ac.jp

Phone: 0081-3-5803-5483
Introduction: Due to its ability to arrest untreated dental caries, silver diamine fluoride (SDF) has been advocated for indirect pulp capping procedures. However, the high concentrations of silver and fluoride in SDF raise concerns about its biocompatibility to pulpal tissues. Objectives: This study aimed to investigate the effect of SDF on the viability, alkaline phosphatase (ALP) activity, and morphology of pulpal-like cells (RPC-C2A) and to evaluate the influence of reduced glutathione (GSH) on SDF-induced cytotoxicity and deposit formation on dentin. Methodology: The cytotoxicity of diluted $38 \%$ SDF solutions (10-4 and 10-5), with or without the addition of $5 \mathrm{mM}$ or $50 \mathrm{mM} \mathrm{GSH}$, was evaluated at 6 and 24 hours. Cell viability was detected using WST-8 and the effect on ALP activity was performed using an ALP assay kit. Cell morphology was observed using a phase-contrast microscope. Scanning electron microscopy analysis was conducted to evaluate the effect of GSH incorporation or conditioning on SDF-induced deposit formation on dentin discs. Cytotoxicity data were analyzed by two-way analysis of variance (ANOVA) and Tukey post hoc tests $(p<0.05)$. Results: There were significant differences between the groups. The results demonstrated that all tested SDF dilutions caused a remarkable cytotoxic effect, while the addition of GSH prevented SDF-induced damage at 6-hour exposure time in the higher dilution of SDF. Dentin treated with plain SDF or GSH-incorporated SDF solution showed deposit formation with occluded dentinal tubules, unlike the other groups. Conclusion: SDF severely disturbed the viability, mineralization-ability, and morphology of pulpal-like cells, while controlled concentrations of GSH had a short-term protective effect against SDF-induced damage. GSH showed an inhibitory effect on SDF-induced dentinal deposit formation. Further research is warranted to evaluate the effect of GSH on caries-arresting, anti-hypersensitivity, and antibacterial functions of SDF.

Keywords: Cytotoxicity. Glutathione. Pulp cells. Silver diamine fluoride.

${ }^{1}$ Tokyo Medical and Dental University, Graduate School of Medical and Dental Sciences, Department of Cariology and Operative Dentistry, Tokyo, Japan.

'University of Sharjah, College of Dental Medicine, Department of Preventive and Restorative Dentistry, Sharjah, United Arab Emirates (UAE)

${ }^{3}$ Tokyo Medical and Dental University, Bio-Matrix (Dental Pharmacology), Tokyo, Japan.

${ }^{4}$ National Guard Health Affairs, King Saud bin Abdulaziz University for Health Sciences, College of

Dentistry, Restorative and Prosthetic Dental Sciences, Riyadh, Saudi Arabia.

${ }^{5}$ National Guard Health Affairs, King Abdullah International Medical Research Centre, Riyadh, Saudi Arabia.

${ }^{6}$ Asahi University, School of Dentistry, Division of Oral Functional Science and Rehabilitation,

Department of Operative Dentistry, Gifu, Japan. 


\section{Introduction}

Silver diamine fluoride (SDF), an alkaline colorless solution composed of $24-29 \%$ silver $(\mathrm{Ag})$ and $5-6 \%$ fluoride $(F)$, is used for arresting caries progression and treating dentin hypersensitivity. This agent is gaining considerable attention due to the promising results of both clinical and laboratory studies. ${ }^{1}$ The mechanisms of action are still not clearly understood. However, it is believed that $\mathrm{Ag}$ ions provide antibacterial properties against some cariogenic bacteria, and $F$ ions enhance remineralization. ${ }^{2,3}$ SDF also forms insoluble precipitates with calcium and phosphate that physically block dentinal tubules, minimizing dentin hypersensitivity. ${ }^{4}$ SDF application is considered a simple, inexpensive, and non-invasive procedure. Nevertheless, it can lead to discoloration of teeth. ${ }^{5}$ The $\mathrm{Ag}$ ions can infiltrate within demineralized and the underlying mineralized dentin. ${ }^{6} \mathrm{Ag}$ and $\mathrm{F}$ ions can penetrate up to $0.2 \mathrm{~mm}$ into dentin. ${ }^{7}$ Despite the popularity of SDF, some reports suggest a cautious application of this solution into the oral cavity. ${ }^{8,9}$ Compared to silver chloride, SDF has higher toxicity against osteoblast-like cells and human gingival fibroblasts (HGF) - only one hour of contact with HGF induced irreversible cell death by necrosis. ${ }^{8} \mathrm{~F}$ concentration $(44,800 \mathrm{ppm})$ in SDF is considered one of the highest compared to other available products. ${ }^{10}$ The high concentrations of $\mathrm{Ag}$ and $\mathrm{F}$ raise concerns about their harmful effects on pulpal tissue. The cytotoxic effect of SDF on HGF occurred even at low concentrations $(0.1 \%)$ and lasted for nine weeks even after rinsing SDF-treated dentin discs with saline. ${ }^{9}$ Despite inadequate investigations on the cytotoxic effect of SDF on pulpal cells, studies show the potential use of SDF in indirect pulp capping procedure in deep cavities and gained much attention due to SDF's ability to induce tertiary dentin formation. ${ }^{11}$

Glutathione is a low molecular weight thiolcompound that contains a sulfhydryl group in its structure. It has an essential role as an antioxidant that functions by various mechanisms such as metal chelators and radical quenchers. ${ }^{12,13}$ Glutathione is possibly the most prevalent and most important intracellular thiol-disulfide redox buffer in mammalian cells. The active form of glutathione is called reduced glutathione (GSH), a water-soluble tripeptide containing cysteine, glutamic acid, and glycine that contributes to the intracellular non-protein thiols. ${ }^{14-16} \mathrm{Ag}$ cytotoxicity on rat and human liver cells could partially result from GSH depletion. ${ }^{17,18}$ The strong affinity of $\mathrm{Ag}$ to the sulfhydryl group could be responsible for the observed GSH depletion. ${ }^{19}$ Moreover, GSH is involved in the detoxification of $\mathrm{Ag}$ from blood plasma. ${ }^{20} \mathrm{~F}$ is also known to decrease the level of GSH, which reflects increased utilization of the latter due to the oxidative stress generated by the administration of $\mathrm{F}$ at a level of $25 \mathrm{ppm}$ in the drinking water of rats. ${ }^{21}$ The increased oxidative stress and the adverse effect of $F$ on the antioxidant function have also been confirmed in several other studies. ${ }^{22-26}$

There is a paucity of scholarly literature on the adverse effect of SDF on pulpal cells and preventing or minimizing this damage. Thus, this study aims to assess the effect of SDF on rat-pulpal cells, test the effect of GSH on SDF-induced damage on the cells as mentioned earlier, and its effect on the ability of SDF to form deposits on the surface of dentin discs. The tested null hypotheses were that: (i) SDF has no toxic effect on pulpal-like cells, (ii) GSH does not counteract the adverse effect of SDF on pulpal-like cells, and (iii) GSH does not affect SDF-induced deposit formation on dentin surfaces.

\section{Methodology}

\section{Effect on cell proliferation, ALP activity, and morphology}

\section{Cell proliferation assay}

The clonal cell line (RPC-C2A), pulp-like cells, was used in this study. ${ }^{27}$ To each well of 24-well culture plates, RPC-C2A cells $\left(5 \times 10^{4}\right.$ cells/well) were placed and incubated for $24 \mathrm{~h}$ in a $5 \% \mathrm{CO}_{2}$ incubator at $37^{\circ} \mathrm{C}$. Six wells were allocated for each test solution for an observation time of 6 and $24 \mathrm{~h}$. The test solutions included two dilutions of $38 \% \operatorname{SDF}(1,000$ and 10,000$)$ (Bee Brand Medico Dental, Osaka, Japan) with or without 50 or $500 \mathrm{mM}$ of GSH (L-Glutathione reduced, Sigma Aldrich Co., St. Louis, MO, USA). The prepared solutions were further diluted in a cell culture medium that contained $10 \%$ FBS in DMEM (Sigma-Aldrich, MO, USA), at a ratio of $1: 9$ (solution:medium).

Using a one-way ANOVA test, the sample size was calculated based on a significance level of $5 \%$ and power of $80 \%$ to detect a difference of 0.2 in spectrophotometric absorbance. The required sample size for each group is at least 4 (powerandsamplesize. 
com).

The resultant experimental solutions were as the following $(n=6)$ : (I) $\mathrm{SDF}_{x} 10^{-4}(\mathrm{pH} 8.2)$, (II) $\mathrm{SDF}_{x} 10^{-5}$ (pH 8.11), (III) $\mathrm{SDF}_{\mathrm{x}} 10^{-4}+5 \mathrm{mM}$ GSH (pH 7.6), (IV) $\mathrm{SDF}_{\mathrm{x}} 10^{-4}+50 \mathrm{mM} \mathrm{GSH}(\mathrm{pH} 6.12),(\mathrm{V}) \mathrm{SDF}_{\mathrm{x}} 10^{-5}+5$ $\mathrm{mM}$ GSH (pH 7.58), and (VI) $\mathrm{SDF}_{x} 10^{-5}+50 \mathrm{mM} \mathrm{GSH}$ $(\mathrm{pH} 6.3)$. Because of the strong alkaline property of SDF, we diluted SDF to a concentration equal or lower than $\mathrm{SDF}_{\mathrm{x}} 10^{-4}$ to have a $\mathrm{pH}$ less than 8.0, which caused no precipitation or turbidity in the cell culture medium. Cell culture in fresh medium $(\mathrm{pH}$ 7.74) without experimental solution served as the control. After the incubation time, the culture medium was discarded, and cells were washed with $200 \mu \mathrm{L}$ phosphate buffer solution to prevent any interaction between the test solutions and the colorimetric assay. One hundred microliters of new culture medium were added to each well, and cell viability was measured using 2-(2-Methoxy-4-nitrophenyl)-3-(4-nitrophenyl)5-(2,4-disulfophenyl)-2H-tetrazolium, mono-sodium salt [WST-8] (Cell Counting kit-8; Dojindo, Tokyo, Japan), which depends on the ability of mitochondrial dehydrogenases to oxidize WST- 8 into a soluble, purple formazan. The spectrophotometric absorbance (optical density [OD]) of the samples at $450 \mathrm{~nm}$ was measured using a microplate reader. A blank well was regularly used for data subtraction by placing the same volume of culture medium with WST- 8 solution into culture wells. The morphology of the cultured cells was observed by using a phase-contrast microscope (1X70; Olympus, Tokyo, Japan).

\section{ALP activity measurement}

Cultured RPC-C2A cells were treated with the same solutions described above for 6 or $24 h(n=6)$. ALP activity was determined by using ALP Assay Kit (Takara Bio, Shiga, Japan).

\section{Scanning electron microscopy analysis of dentin discs treated with SDF}

According to the protocol approved by the Human Research Ethics Committee, extracted human noncarious third molars were used in this part of the study. Flat dentin discs were created perpendicular to the tooth's longitudinal axis, using a slow-speed diamond saw (Isomet Low Speed Saw; Buehler, Lake Bluff, IL, USA) under water lubrication. Dentin surfaces were polished using up to 4000 -grit silicon-carbide paper under water irrigation, followed by sonication with deionized water for debris removal. The dentin discs were randomly divided into four groups. In group 1 (control group), dentin discs were treated with distilled water. In groups 2 and 3, 38\% SDF (pH 10) was applied, followed by either distilled water or $20 \%$ GSH solution ( $\mathrm{pH} 4$ ), respectively. Group 4 discs were treated with a solution that contained $38 \%$ SDF and $20 \% \mathrm{GSH}$ ( $\mathrm{pH} 6.8-7.8$ ). All solutions were applied with agitation using micro-brushes for $1 \mathrm{~min}$. A final rinse with water for $30 \mathrm{~s}$ was performed on all dentin discs. Specimens were stored in a simulated body fluid (SBF) of a $7.4 \mathrm{pH}$ for $6 \mathrm{~h}$. SBF solution was prepared by dissolving $136.8 \mathrm{mM} \mathrm{NaCl}, 4.2 \mathrm{mM} \mathrm{NHCO}_{3}, 3 \mathrm{mM} \mathrm{KCl}$, $1 \mathrm{mM} \mathrm{K}_{2} \mathrm{HPO}_{4} \cdot 3 \mathrm{H}_{2} \mathrm{O}, 1.5 \mathrm{mM} \mathrm{MgCl}$ and $0.5 \mathrm{mM} \mathrm{Na}_{2} \mathrm{SO}_{4}$ in water. ${ }^{28}$ Specimens were dried inside a covered glass vial, sputter-coated with gold/palladium, and then observed under a scanning electron microscope (SEM) (JSM-IT100 scanning microscope; JEOL, Tokyo, Japan) operating at $20 \mathrm{kV}$.

\section{Statistical Analysis}

Statistical analysis was performed by applying a two-way analysis of variance (ANOVA) and Tukey's post hoc test using the experimental solution and exposure time as two factors. The analysis was performed using SPSS (IBM SPSS Statistics, v21; IBM Corp.) with a significance level set at $5 \%$.

\section{Results}

\section{Effect on viability, ALP activity, and morphology of RPC-C2A cells}

Figure 1 shows the effect of the tested solutions on the viability of RPC-C2A cells after 6 or $24 \mathrm{~h}$ of exposure. At both exposure times, the two dilutions of SDF showed lower OD values than the control group $(\mathrm{p}<0.001)$. At $6 \mathrm{~h}, \mathrm{SDF}_{\mathrm{x}} 10^{-4}+5 \mathrm{mM} \mathrm{GSH}$ and $\mathrm{SDF}_{\mathrm{x}} 10^{-4}+50 \mathrm{mM}$ GSH solutions had OD values that were statistically higher than both dilutions of SDF without GSH $(p<0.001)$; however, these values were statistically significantly lower than the control group $(p<0.001)$. Meanwhile, there was no statistically significant difference among $\operatorname{SDF}_{\mathrm{x}} 10^{-5}, \mathrm{SDF}_{\mathrm{x}} 10^{-4}$, $\mathrm{SDF}_{\mathrm{x}} 10^{-4}+5 \mathrm{mM} \mathrm{GSH}$ and $\mathrm{SDF}_{\mathrm{x}} 10^{-4}+50 \mathrm{mM}$ GSH at 24 $h(p=0.628)$. At both exposure times, the OD values obtained in $\mathrm{SDF}_{\mathrm{x}} 10^{-5}+5 \mathrm{mM}$ GSH and $\mathrm{SDF}_{\mathrm{x}} 10^{-5}+50$ mM GSH solutions were statistically higher than both dilutions of SDF without GSH $(p<0.001)$. At 6 h, SDF 10 ${ }^{5}+5 \mathrm{mM} \mathrm{GSH}$ and $\mathrm{SDF}_{x} 10^{-5}+50 \mathrm{mM}$ GSH solutions had 
$\mathrm{OD}$ values that were comparable to the control group $(p=0.113)$; however, at $24 \mathrm{~h}$, the resultant OD values were statistically significantly lower than the control group $(p<0.001)$. At both exposure times, $\mathrm{SDF}_{\mathrm{x}} 10^{-5}+5$ $\mathrm{mM}$ GSH and $\mathrm{SDF}_{\mathrm{x}} 10^{-5}+50 \mathrm{mM}$ GSH solutions had OD values that were statistically significantly higher than all other experimental solutions of the same exposure time $(p<0.001)$.

Figure 2 shows the effect of the tested solutions on the ALP activity of RPC-C2A cells after 6 or $24 \mathrm{~h}$ of exposure. At both exposure times, the two plain dilutions of SDF showed statistically significantly lower ALP activity than the control group $(p<0.001)$. All solutions containing GSH had ALP activities that were statistically significantly higher than the two plain dilutions of SDF, at both exposure times $(p<0.001)$. At 6-h exposure time, all solutions containing GSH had ALP activities that were lower than the control $(\mathrm{p}<0.001)$, while at 24-h exposure time, $\mathrm{SDF}_{\mathrm{x}} 10^{-5}+$ $50 \mathrm{mM}$ GSH group showed an ALP activity that is comparable to the control $(p=0.727)$.

Figure 3 shows cell morphology at $24 \mathrm{~h}$ of all groups. Morphologically, RPC-C2A cells in the control media showed polygonal appearance (Figure 3a), whereas cells treated with $\operatorname{SDF}_{x} 10^{-4}$ (Figure $3 \mathrm{~b}$ ) or $\mathrm{SDF}_{\mathrm{x}} 10^{-5}$ (Figure $3 \mathrm{c}$ ) exhibited contracted and spherical morphology with increased intercellular spaces suggestive of cellular death and decreased proliferation. At $6 \mathrm{~h}$, most of the cells in the GSHcontaining groups exhibited normal polygonal

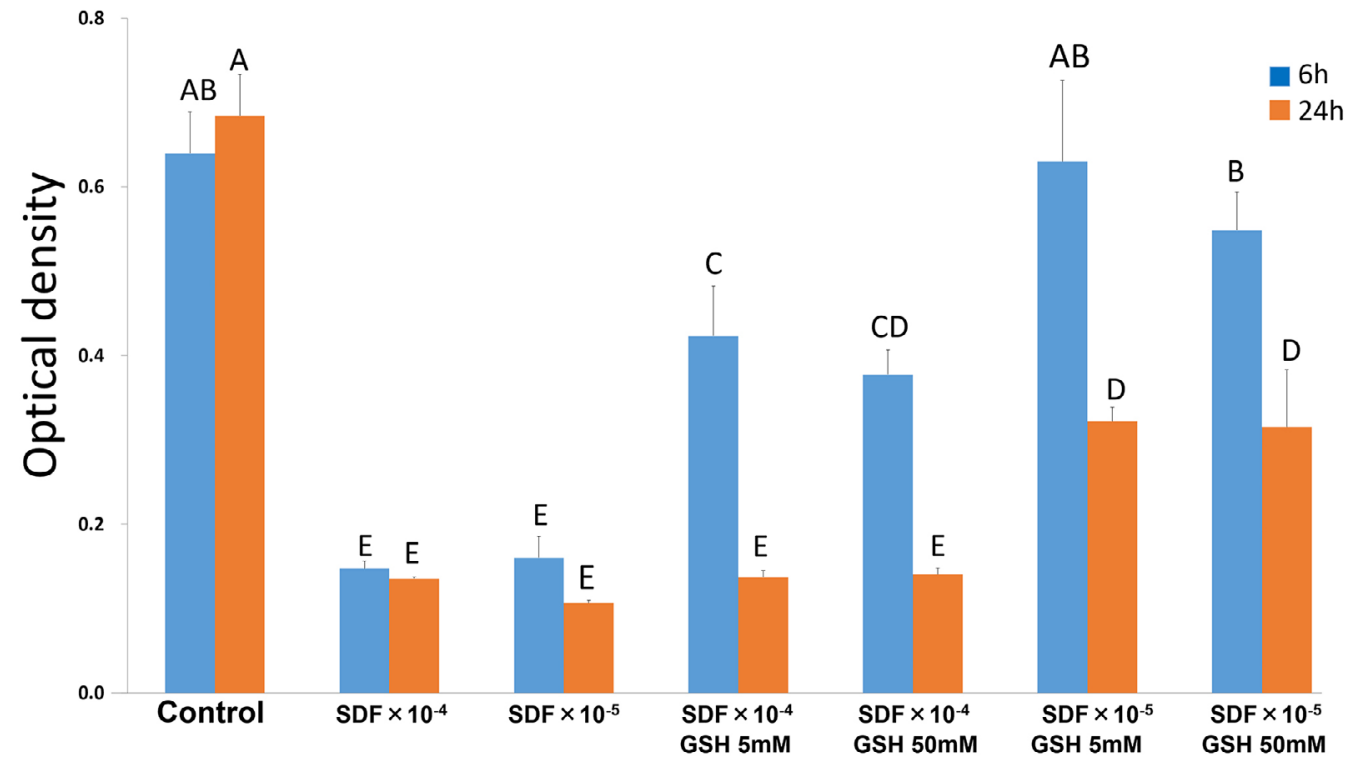

Figure 1- Cytotoxicity of culture medium on RPC-C2A cells containing diluted SDF with and without GSH after 6 and $24 \mathrm{~h}$ of incubation. Cell viability was determined by using WST-8 assay. Data were analysed by using two-way ANOVA. The same letter indicates no significant differ-ence $(P>.05)$

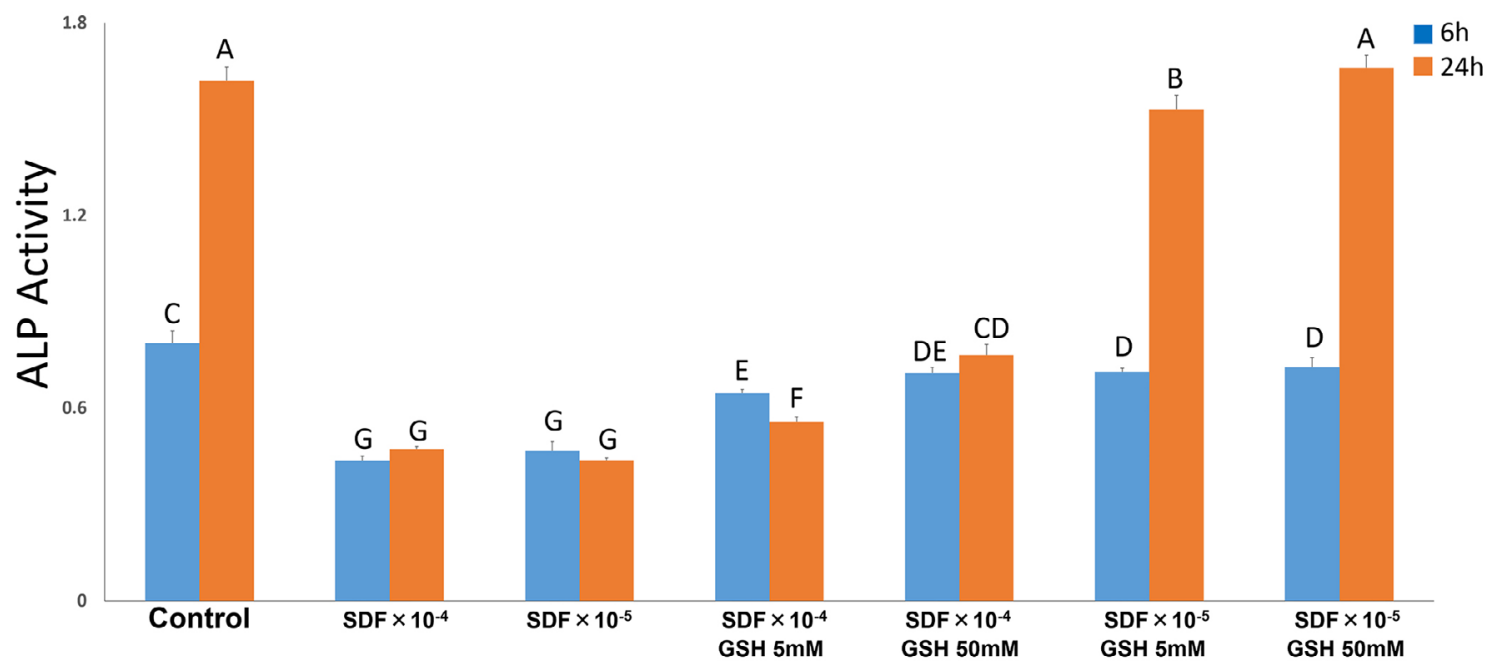

Figure 2- ALP activity of RPC-C2A cells cultured with diluted of SDF with and without GSH for 6 and $24 \mathrm{~h}$. Data were analysed by using two-way ANOVA. letter indicates no significant differ-ence $(P>.05)$ 


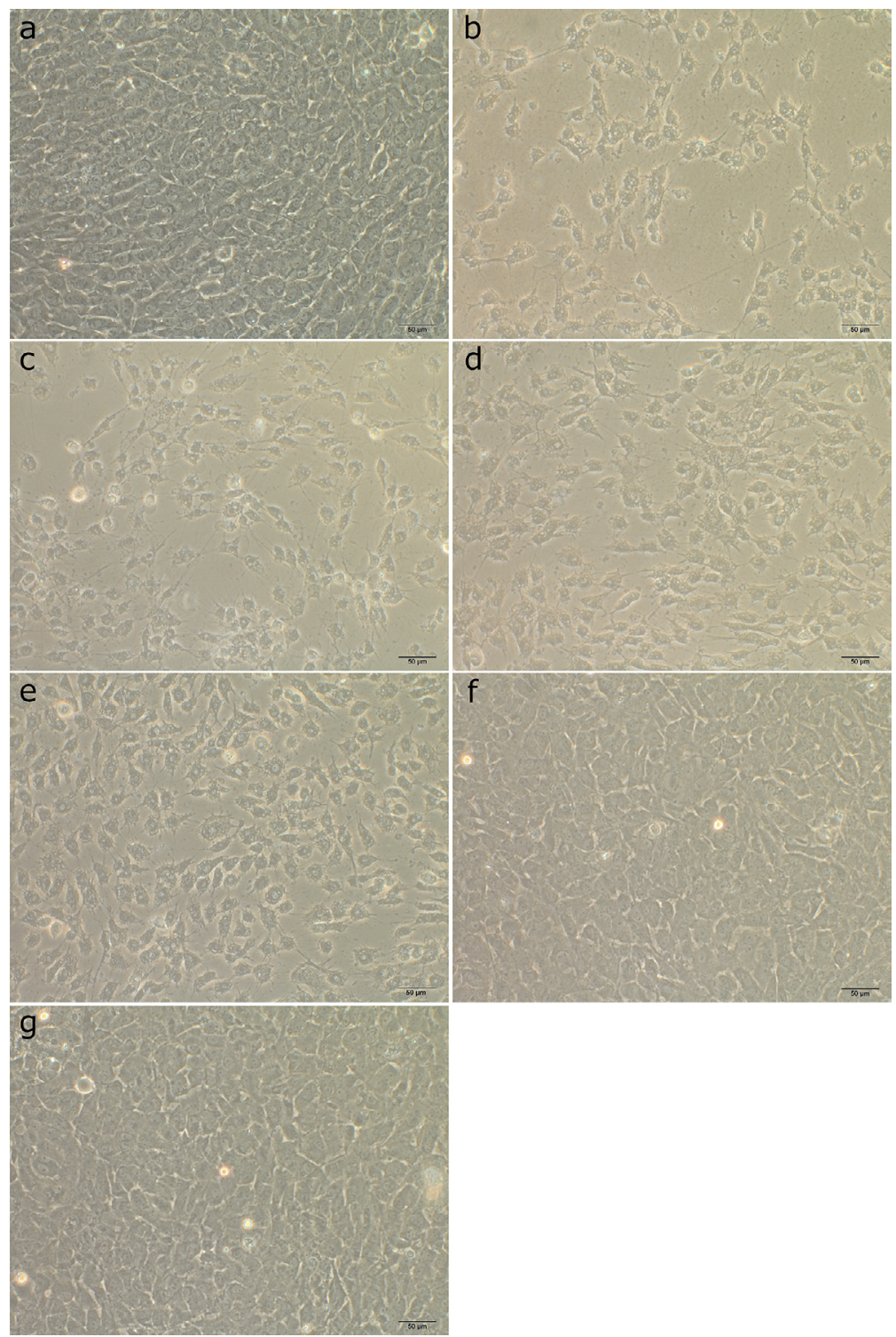

Figure 3- Morphologic changes of RPC-C2A cells after $24 \mathrm{~h}$ of exposure to test solutions. (a) Polygonal-shaped cells of the control group. (b-e) Cells treated with SDFX10-4, SDFX10-5, SDFX10-4+5 mM GSH, or SDFX10-4+50 mM GSH, respectively, showed contracted and spheri-cal morphology and increases in intercellular spaces were observed. ( $f$ and $g$ ) Cells treated with SDFX10-5+5 mM GSH or SDFX10-5+50 mM GSH, respectively, exhibited mostly normal polygo-nal morphology

morphology (data not shown); however, at $24 \mathrm{~h}$, decreased cellular density and increased intercellular spaces for both GSH concentrations with SDFx10-4 (Figure 3d and e) were observed while these changes were less evident in the groups containing GSH with SDFx $10^{-5}$ (Figure $3 f$ and $g$ ) where most of the cells showed normal morphology.

\section{SEM analysis of dentin discs treated with SDF}

Dentin discs treated with $38 \%$ SDF followed by a rinse with distilled water showed marked mineral deposit formation on the surface along with occluded dentinal tubules (Figure 4b). Nevertheless, these findings were less prominent on dentinal surfaces treated with the solution that contained $38 \%$ SDF and $20 \% \mathrm{GSH}$, followed by a rinse with distilled water
(Figure 4c). Meanwhile, dentin discs treated with 38\% SDF followed by conditioning with $20 \%$ GSH showed less deposit formation than the aforementioned experimental groups (Figure 4d). The control group showed the least amount of deposit formation (Figure 4a).

\section{Discussion}

This study is the first to assess the effect of SDF on pulpal-like cells and the efficacy of GSH to mitigate SDF-induced toxicity. We rejected the null hypotheses because SDF adversely affected the viability, ALP activity and morphology of the used cells, and GSH at specific concentrations and exposure time mitigated 


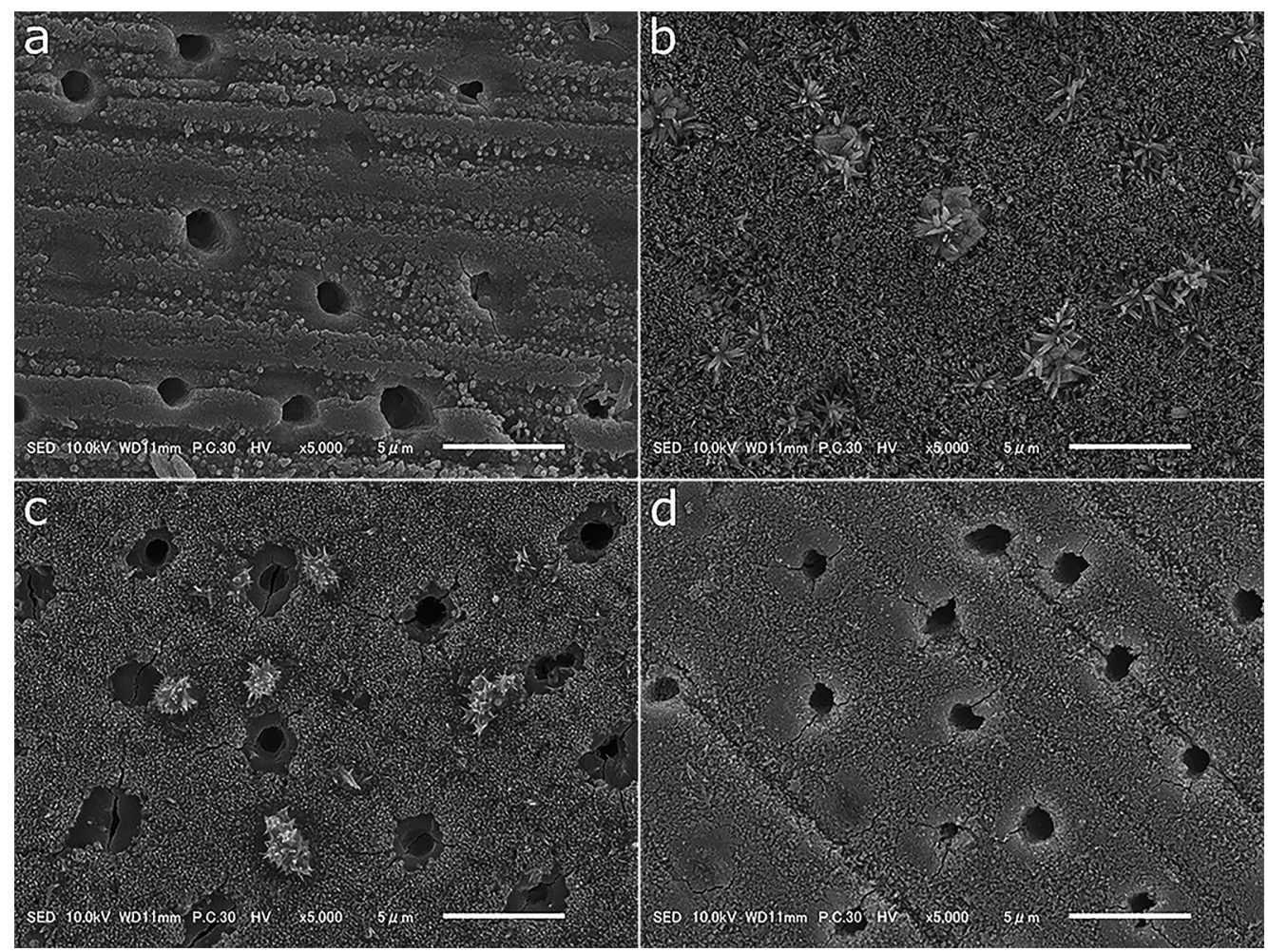

Figure 4- Topographical scanning electron microscopy images of flat dentinal surfaces treated with different solutions. (a) control, (b) $38 \%$ SDF followed by distilled water, (c) $38 \%$ SDF incor-porated with $20 \%$ GSH followed by distilled water, and (d) $38 \%$ SDF followed by $20 \%$ GSH ap-plication

SDF-induced toxicity and negatively affected SDFinduced deposit formation on dentin.

One of the recently suggested SDF applications is its use in deep cavities for indirect pulp capping procedures due to the claimed ability of SDF to induce tertiary dentin formation. ${ }^{11}$ Despite the established safety of SDF application in this procedure, there is always a concern about the effect of the released $F$ and $\mathrm{Ag}$ ions $\mathrm{s}^{8,9}$, which can penetrate easily into dentin, especially within demineralized dentin left at the deepest part of the cavity preparation in indirect pulp procedures, ${ }^{6,7}$ thus the need for a non-toxic indirect pulp capping agent cannot be stressed enough. Due to the chances of pulpal cells exposure to SDF, pulpal-like cells were used in this study to assess its effect on the viability, ALP activity, and morphology of these cells. In a preliminary study, we used higher SDF concentrations in the cell culture medium than the concentrations used in this study. We noticed white turbidity and increased $\mathrm{pH}(>8)$ of cell culture medium at high SDF concentrations. Thus, in this study, $38 \%$ SDF was diluted at 10,000-fold (0.0038\%) and 100,000 -fold $(0.00038 \%)$, which showed no turbidity in the culture medium. SDF at $0.0038 \%$ and $0.00038 \%$ adversely affected cell viability and changed cellular morphology from polygonal to round with loss of intercellular spaces. SDF also suppressed the ALP activity of the cells. ALP activity correlates with the mineralization ability of the cells. ${ }^{29}$ Thus, it is essential to maintain this activity for successful indirect pulp therapy procedure by the formation of tertiary dentin. It was reported that the cytotoxicity of SDF is related to both $\mathrm{F}$ and $\mathrm{Ag}$ constituents with a synergistic interaction between these two constituents that heightens the cellular damage ${ }^{8}$ and enhances oxidative stress. ${ }^{30}$ The mechanism of this enhanced toxic effect of $\mathrm{Ag}$ and $\mathrm{F}$ is speculated to depend on the increased generation of reactive oxygen species (ROS) or lipid peroxidation accompanied by a reduction in the total antioxidant capacity leading to cell death and inflammation. ${ }^{30}$ In this study, the supplementation of the experimental solutions with 5 $\mathrm{mM}$ or $50 \mathrm{mM} \mathrm{GSH}$ led to a decreased toxic effect of both dilutions of SDF at 6-h exposure time; however, this protective effect was lost at 24-h exposure time for both GSH concentrations with $0.0038 \%$ SDF. This confirms the finding that SDF has a long-standing effect even at low concentration, which is beneficial for its antimicrobial application; nonetheless, it also raises concerns about its cytotoxicity. ${ }^{9}$ This prolonged effect is referred to as the "zombie effect", where dead cells act as a reservoir of Ag, further affecting healthy nearby cells. ${ }^{31}$ As stated earlier, both $\mathrm{Ag}$ and $\mathrm{F}$ cause depletion in the level of $\mathrm{GSH}^{19,21}$ thus, several 
mechanisms can be proposed for the action of GSH in protecting pulpal cells from SDF-induced damage: (i) enhancement of cellular levels of $\mathrm{GSH}, 32$ (ii) reduction of ROS levels; ${ }^{33}$ and/or (iii) direct interaction of GSH with $\mathrm{Ag}$ resulting in the formation of complexes that help in detoxification of $\mathrm{Ag} .^{20}$

We assessed the effect of GSH on SDF-induced deposit formation, and the results showed that applying $20 \% \mathrm{GSH}$ as a separate step after SDF significantly decreased the formation of these deposits with several open dentinal tubules. However, when GSH was incorporated within SDF, this inhibitory effect on deposit formation was less pronounced. GSH was previously studied for its ability to decrease SDF-induced color changes of tooth structure. ${ }^{34}$ The process through which GSH affects the function of SDF is still unclear; however, the interaction with $\mathrm{Ag}$ and/ or the acidity of GSH might have contributed to the current findings. The demineralizing effect of a low $\mathrm{pH}$ agent was suggested to harm the stability of crystal precipitations on dentin surfaces, ${ }^{35}$ and this might be true for the group where $20 \%$ GSH was applied separately on dentin after SDF application. Both F and $\mathrm{Ag}$ ions contribute to the formation of SDF-induced precipitates. ${ }^{36,37}$ Significant Ag layer accumulation is reported on dentin surfaces treated with SDF. ${ }^{38} \mathrm{GSH}$ is known to interact with silver, ${ }^{19,20,39}$ and we speculate that the formation of these complexes could minimize the action of $\mathrm{Ag}$ in forming crystals on dentin surfaces.

\section{Conclusion}

The limitations of this study include, but are not limited to, the utilization of extracted human teeth from different subjects and the use of in vitro experimental conditions that contribute to only limited answers to more complex problems. Within these limitations, SDF showed detrimental effects on the viability, ALP activity, and morphology of the pulpal-like cells. The use of GSH at specific concentrations and exposure times attenuated the toxic effect of SDF; however, it negatively affected SDF-induced deposit formation on dentin. This protective effect of GSH may provide insights into the development of a "smart," more biocompatible agent. Further research could assess the repercussions of GSH application on caries-arresting, anti-hypersensitivity, and antibacterial functions of SDF using in vivo animal models.

\section{Author disclosure statement}

The authors declare no conflicts of interest.

\section{Authors' contributions}

Kim, Seunggun: Data curation (Equal); Investigation (Equal); Methodology (Equal); Writing-original draft (Equal). Nassar, Mohannad: Conceptualization (Equal); Formal analysis (Equal); Software (Equal); Writing-original draft (Equal); Writing-review \& editing (Equal). Tamura, Yukihiko: Data curation (Equal); Investigation (Equal); Methodology (Equal); Validation (Equal); Visualization (Equal); Writing-review \& editing (Equal). Hiraishi, Noriko: Conceptualization (Equal); Investigation (Equal); Project administration (Equal); Resources (Equal); Supervision (Equal); Validation (Equal); Writing-original draft (Equal); Writing-review \& editing (Equal). Jamleh, Ahmed: Formal analysis (Equal); Software (Equal); Writing-original draft (Equal); Writing-review \& editing (Equal). Nikaido, Toru: Supervision (Equal); Visualization (Equal); Writingreview \& editing (Equal). Tagami, Junji: Project administration (Equal); Supervision (Equal); Writingreview \& editing (Equal).

\section{References}

1- Mei ML, Lo EC, Chu CH. Arresting dentine caries with silver diamine fluoride: what's behind it? J Dent Res. 2018; 97(7):751-8. doi: $10.1177 / 0022034518774783$

2- Vasquez E, Zegarra G, Chirinos E, Castillo JL, Taves DR, Watson $\mathrm{GE}$, et al. Short term serum pharmacokinetics of diammine silver fluoride after oral application. BMC Oral Health. 2012;12:60. doi: 10.1186/1472-6831-12-60

3- Nyvad B, ten Cate JM, Fejerskov O. Arrest of root surface caries in situ. J Dent Res. 1997;76(12):1845-53. doi: 10.1177/00220345970760120701

4- Castillo JL, Rivera S, Aparicio T, Lazo R, Aw TC, Mancl LL, et al. The short-term effects of di-ammine silver fluoride on tooth sensitivity: a randomized controlled trial. J Dent Res. 2011;90(2):203-8. doi: $10.1177 / 0022034510388516$

5- Horst JA, Ellenikiotis H, Milgrom PL. UCSF protocol for caries arrest using silver diamine fluo-ride: rationale, indications and consent. J Calif Dent Assoc. 2016;44(1):16-28.

6- Sayed M, Matsui N, Uo M, Nikaido T, OIkawa M, Burrow MF, et al. Morphological and elemental analysis of silver penetration into sound/demineralized dentin after SDF application. Dent Mater. 2019;35(12):1718-27. doi: 10.1016/j.dental.2019.08.111

7- $\mathrm{Chu} \mathrm{CH}$, Lo EC. Microhardness of dentine in primary teeth after topical fluoride applications. J Dent.2008;36(6):387-91. doi: 10.1016/j. jdent.2008.02.013

8- Contreras RG, Sakagami H, Nakajima H, Shimada J. Type of cell death induced by various metal cations in cultured human gingival fibroblasts. In Vivo. 2010;24(4):513-7. 
9- Fancher ME, Fournier S, Townsend J, Lallier TE. Cytotoxic effects of silver diamine fluoride. Am J Dent. 2019;32(3):152-6.

10- Mei ML, Chu CH, Lo EC, Samaranayake LP. Fluoride and silver concentrations of silver diam-mine fluoride solutions for dental use. Int J Paediatr Dent. 2013;23(4):279-85. doi: 10.1111/ipd.12005 11- Korwar A, Sharma S, Logani A, Shah N. Pulp response to high fluoride releasing glass ionomer, silver diamine fluoride, and calcium hydroxide used for indirect pulp treatment: an in-vivo comparative study. Contemp Clin Dent. 2015;6(3):288-92. doi: 10.4103/0976237X.161855

12- Mulier B, Rahman I, Watchorn T, Donaldson K, Macnee W, Jeffery PK. Hydrogen peroxide-induced epithelial injury: the protective role of intracellular nonprotein thiols (NPSH). Eur Respir J. 1998;11(2):38491. doi: 10.1183/09031936.98.11020384

13- Deneke SM. Thiol-based antioxidants. Curr Top Cell Regul 2000;36:151-80. doi: 10.1016/s0070-2137(01)80007-8

14- Meister A, Anderson ME. Glutathione. Annu Rev Biochem. 1983;52:711-60. doi: 10.1146/annurev.bi.52.070183.003431

15- Pompella A, Visvikis A, Paolicchi A, De Tata V, Casini AF. The changing faces of glutathione, a cel-lular protagonist. Biochem Pharmacol. 2003;66(8):1499-503. doi: 10.1016/s0006-2952(03)00504-5

16- Jacobson JM, Michael JR, Jafri MH Jr, Gurtner GH. Antioxidants and antioxidant enzymes protect against pulmonary oxygen toxicity in the rabbit. J Appl Physiol (1985). 1990;68(3):1252-9. doi: 10.1152/ jappl.1990.68.3.1252

17- Piao MJ, Kang KA, Lee IK, Kim HS, Kim S, Choi JY, et al. Silver nanoparticles induce oxidative cell damage in human liver cells through inhibition of reduced glutathione and induction of mitochondriainvolved apoptosis. Toxicol Lett. 2011;201(1):92-100. doi: 10.1016/j. toxlet.2010.12.010

18- Hussain SM, Hess KL, Gearhart JM, Geiss KT, Schlager JJ. In vitro toxicity of nanoparticles in BRL $3 \mathrm{~A}$ rat liver cells. Toxicol In Vitro. 2005;19(7):975-83. doi: 10.1016/j.tiv.2005.06.034.

19- Kramer J, Bell R, Smith S, Gorsuch J. Silver nanoparticle toxicity and biocides: need for chemical speciation. Integr Environ Assess Manag. 2009;5(4):720-2. doi: 10.1897/IEAM_2009-066.1

20- Khan $\mathrm{H}$, Khan MF, Asim-ur-Rehman, Jan SU, Ullah N. The protective role of glutathione in silver in-duced toxicity in blood components. Pak J Pharm Sci. 2011;24(2):123-8.

21- Shanthakumari D, Srinivasalu S, Subramanian S. Effect of fluoride intoxication on lipidperoxidation and antioxidant status in experimental rats. Toxicology. $2004 ; 204(2-3): 219-28$. doi: $10.1016 / j$. tox.2004.06.058

22- Gong T, Bai CM, Chen T, Peng X, Cui HM. Effect of high fluorine on the antioxidant function and ul-trastructure of liver in chickens $\mathrm{Xu} \mathrm{Mu}$ Shou Yi Xue Bao. 2009;40(9):1389-94. Chinese

23- Chen T, Cui H, Cui Y, Bai C, Gong T, Peng X. Cell-cycle blockage associated with increased apop-totic cells in the thymus of chickens fed on diets high in fluorine. Hum Exp Toxicol. 2011;30(7):685-92. doi: 10.1177/0960327110379022

24- Luo Q, Cui H, Peng X, Fang J, Zuo Z, Deng J, et al. Intestinal IgA+ cell numbers as well as IgA, IgG, and IgM contents correlate with mucosal humoral immunity of broilers during supplementation with high fluorine in the diets. Biol Trace Elem Res. 2013;154(1):62-72. doi: $10.1007 / \mathrm{s} 12011-013-9713-9$
25- Kuang P, Deng $H$, Cui $H$, Chen L, Fang J, Zuo Z, et al. Sodium fluoride (NaF) causes toxic effects on splenic development in mice. Oncotarget. 2017;8(3):4703-17. doi: 10.18632/oncotarget.13971 26- Lu Y, Luo Q, Cui H, Deng H, Kuang P, Liu H, et al. Sodium fluoride causes oxidative stress and apoptosis in the mouse liver. Aging (Albany NY). 2017;9(6):1623-39. doi: 10.18632/aging.101257

27- Kasugai S, Adachi M, Ogura H. Establishment and characterization of a clonal cell line (RPC-C2A) from dental pulp of the rat incisor. Arch Oral Bio. 1988;33(12):887-91. doi: 10.1016/0003-9969(88)90018-0 28- Kokubo T, Takadama $\mathrm{H}$. How useful is SBF in predicting in vivo bone bioactivity? Biomaterials. 2006;27(15):2907-15. doi: 10.1016/j. biomaterials.2006.01.017

29- Beck GR Jr, Sullivan EC, Moran E, Zerler B. Relationship between alkaline phosphatase levels, os-teopontin expression, and mineralization in differentiating MC3T3-E1 osteoblasts. J Cell Biochem. 1998;68(2):269-80. doi: 10.1002/(sici)10974644(19980201)68:2<269: : aid-jcb13>3.0.co;2-a

30- Inkielewicz-Stepniak I, Santos-Martinez MJ, Medina C, Radomski MW. Pharmacological and toxi-cological effects of co-exposure of human gingival fibroblasts to silver nanoparticles and sodium fluoride. Int J Nanomedicine. 2014;9(1):1677-87. doi: 10.2147/IJN.S59172

31- Wakshlak RB-K, Pedahzur R, Avnir D. Antibacterial activity of silver-killed bacteria: the "zombies" effect. Sci Rep. 2015;5:9555. doi: 10.1038/srep09555

32- Yamada M, Kojima N, Att W, Hori N, Suzuki T, Ogawa T. N-Acetyl cysteine restores viability and function of rat odontoblast-like cells impaired by polymethylmethacrylate dental resin extract. Redox Rep. 2009;14(1):13-22. doi: 10.1179/135100009X392430

33- Spagnuolo F, D Anto V, Cosentino C, Schmalz G, Schweikl H, Rengo $\mathrm{S}$. Effect of $\mathrm{N}$-acetyl-L-cysteine on ROS production and cell death caused by HEMA in human primary gingival fibroblasts. Bio-materials. 2006;27(9):1803-09. doi: 10.1016/j.biomaterials.2005.10.022

34- Sayed M, Matsui N, Hiraishi N, Nikaido T, Burrow MF, Tagami J. Effect of glutathione bio-molecule on tooth discoloration associated with silver diammine fluoride. Int J Mol Sci. 2018;19(5):1322. doi: 10.3390/ijms19051322

35- Craig GG, Knight GM, McIntyre JM. Clinical evaluation of diamine silver fluoride/potassium iodide as a dentine desensitizing agent: a pilot study. Aust Dent J. 2012;57(3):308-11. doi: 10.1111/j.18347819.2012.01700.x

36- Thrash WJ, Jones DL, Dodds WJ. Effect of a fluoride solution on dentinal hypersensitivity. Am J Dent. 1992;5(6):299-302.

37- Greenhill JD, Pashley DH. The effects of desensitizing agents on the hydraulic conductance of hu-man dentin in vitro. J Dent Res. 1981;60(3):686-98. doi: 10.1177/00220345810600030401

38- Willershausen I, Schulte D, Azaripour A, Weyer V, Briseño $B$, Willershausen $B$. Penetration poten-tial of a silver diamine fluoride solution on dentin surfaces: an ex vivo study. Clin Lab. 2015;61(11):1695-701. doi: 10.7754/clin.lab.2015.150401 39- Rubino FM. Toxicity of glutathione-binding metals: a review of targets and mechanisms. Toxics. 2015;3(1):20-62. doi: 10.3390/ toxics3010020 